\title{
Zukunft der Arbeit in Industrie 4.0 - Neue Perspektiven und offene Fragen
}

\author{
Alfons Botthof und Ernst Hartmann
}

\section{Perspektiven}

Die technologischen Innovationen, die mit dem Zukunftskonzept ,Industrie 4.0` einhergehen, werden die Arbeitswelt der Zukunft - in der Industrie, aber nicht nur dort - erheblich prägen; in dieser Einschätzung sind sich die Autorinnen und Autoren dieses Bandes einig.

Auch in einigen grundlegenden Fragen, die sich auf das Wesen und die Dynamik dieser Veränderungen beziehen, zeigt sich große Einigkeit.

Zunächst kann als Konsens festgehalten werden, dass die technologischen Innovationen diese Veränderungen in der Arbeitswelt nicht quasi ,naturgesetzlich“ im Sinne eines technologischen Determinismus hervorbringen werden. Die Arbeitswelt der Zukunft unter den Bedingungen von Industrie 4.0 ist gestaltbar und gestaltungsbedürftig.

Diese Gestaltung der Arbeit der Zukunft wird sich paradigmatisch am Konzept des soziotechnischen Systems mit den Dimensionen ,Mensch', ,Organisation “ und ,Technik ‘ müssen. Im Hinblick auf die Gestaltungspraxis kommt dabei der Dimension ,Organisation' besondere Bedeutung zu. Über die inner- und zwischenbetriebliche Organisation werden Wertschöpfungsprozesse realisiert. Die Ausgestaltung der Aufbau- und Ablauforganisation - und insbesondere die damit verbundene Arbeitsteilung und -kombination - ist der wesentliche Einflussfaktor für die Qualität der Arbeit im Hinblick auf die arbeitenden Menschen; zentrale Kriterien der Qualität der Arbeit, wie etwa Persönlichkeitsund Lernförderlichkeit, werden hauptsächlich von solchen organisationalen Parametern bestimmt.

Die Ausgestaltung der Technik sollte sich als Konsequenz daraus orientieren an diesen organisationalen Strukturen und an der Qualität der Arbeit für den Menschen, wobei diese beiden Zieldimensionen wiederum interdependent sind.

\footnotetext{
A. Botthof $(\varangle) \cdot$ E. Hartmann Institut für Innovation und Technik (iit), Berlin, Germany e-mail: botthof@iit-berlin.de 
Weiterhin besteht Konsens dahingehend, dass die Gestaltung der Arbeit der Zukunft Antworten geben muss auf die Herausforderungen des demografischen Wandels. Im Hinblick auf länger andauernde Erwerbsbiografien und zunehmende technologische Dynamik sind es vor allem zwei Aspekte der Arbeitsgestaltung, die hier von besonderer Bedeutung sind:

- Die Entlastung des Menschen von Fehlbeanspruchungen physischer wie psychischer Art und

- die Lernförderlichkeit der Arbeitsprozesse als Voraussetzung für lebenslanges Lernen und dynamische Anpassung an sich verändernde Bedingungen.

Es zeichnen sich auf technologischer Ebene neue Möglichkeiten ab, die sowohl im Hinblick auf organisationale Strukturen und Prozesse wie auch auf die Qualität menschlicher Arbeit neue Gestaltungsmöglichkeiten und -herausforderungen mit sich bringen. Zu diesen Entwicklungen gehören zumindest die folgenden:

- Es werden wesentlich mehr und diversere Informationen - in Echtzeit - zur Verfügung stehen. Die - organisationale - Gestaltungsherausforderung bezieht sich hier darauf, wem diese Informationen für welche Aufgaben und Entscheidungen wie zur Verfügung gestellt werden sollen.

- Der Terminus , autonome Systeme“ bezieht sich auf eine neue Qualität der Automatisierung, die auch die komplexe Wahrnehmung der Realität, anspruchsvolle Analysen und Entscheidungsprozesse mit einbezieht. Diese autonomen Funktionen können im Hinblick auf menschliche Arbeit unterstützende (Assistenzsysteme) und ersetzende Wirkung haben, wobei unterstützende und ersetzende Wirkungen in vielfältigen Mischformen, auch differenziert nach Beschäftigtengruppen, auftreten können.

- Im Bereich der Mensch-Technik-Interaktion zeichnen sich zumindest zwei bedeutsame Trends ab. Der eine dieser Trends ist die mit Begriffen wie Virtualisierung und Augmentierung bezeichnete Verschränkung und Integration natürlicher und virtueller Realitäten. Dies weist weit über traditionelle Konzepte der Mensch-Technik-Interaktion hinaus und erfordert neue Beschreibungs- und Gestaltungskonzepte.

- Insbesondere in der Robotik finden sich erste Realisierungen von Formen der MenschTechnik-Kollaboration und -Kooperation, die ebenfalls über eine bloße ,Nutzung ' oder Interaktion von Technik durch Menschen hinausweisen. Auch hier sind grundlegend und möglicherweise paradigmatisch neue Beschreibungs- und Gestaltungskonzepte erforderlich.

\section{Offene Fragen}

Auf der Grundlage dieser Einschätzungen hinsichtlich prinzipieller Gestaltungsfragen und absehbarer technischer Optionen stellen sich Forschungsfragen in verschiedenen Gegenstandsbereichen. Zu diesen Fragen gehören die folgenden: 
- Welche Herausforderungen und Gestaltungsoptionen ergeben sich hinsichtlich höher qualifizierter Arbeit? Inwieweit werden anspruchsvolle planende und organisierende Tätigkeiten automatisierbar?

- Welche Herausforderungen und Gestaltungsoptionen ergeben sich hinsichtlich geringer qualifizierter Arbeit? Inwieweit und in welchem Umfang werden Tätigkeiten mit geringeren Qualifikationsanforderungen durch autonome technische Systeme übernommen?

- Welche Rolle können Assistenzsysteme spielen, insbesondere auch differenziert nach Anspruchsgehalt der jeweiligen Tätigkeiten und Prozesse? Inwieweit und wie können Assistenzsysteme bei anspruchsvollen Tätigkeiten menschliche Experten in ihrer Expertise - und der weiteren Entwicklung ihrer Expertise - unterstützen? Inwieweit und wie können Assistenzsysteme auch anspruchsvollere Tätigkeiten für Personen mit geringerem Qualifikationsniveau oder Leistungsminderungen beherrschbar machen?

- Wie genau soll die Qualifikations- und Kompetenzentwicklung in der und für die Arbeit umgesetzt werden? In welchem Verhältnis stehen dabei Modelle des Lernens in der Arbeit unmittelbar - lernförderliche Arbeitsorganisation - und arbeitsnahe Formen des Lernens, wie etwa Lernfabriken?

- Wie können autonome technische Systeme mit menschlicher Autonomie in Einklang gebracht werden? Wie stellt man auch in hochautomatisierten Umgebungen menschliche Kontrolle - Kontrolle des Menschen über seine Umwelt und die dort ablaufenden Prozesse - her?

- Welche Gestaltungsmethoden und -methodologien stehen für die Gestaltung der Arbeit in der Industrie 4.0 zur Verfügung? Welche Rolle können dabei Ansätze wie das Ecological Interface Design spielen?

Open Access This chapter is distributed under the terms of the Creative Commons Attribution Noncommercial License, which permits any noncommercial use, distribution, and reproduction in any medium, provided the original author(s) and source are credited. 